Usages du français et pratiques d'enseignement en Europe balkanique, centrale et orientale - Grèce,

Serbie, Bulgarie, Moldavie, Hongrie, Allemagne, Russie - XVIIIe - XXe siècles

\title{
Jean Fleury (1864-1892), auteur d'une grammaire française originale pour les Russes
}

\section{Sergueï Vlassov}

\section{Q OpenEdition \\ Journals}

Édition électronique

URL : https://journals.openedition.org/dhfles/4148

DOI : $10.4000 /$ dhfles.4148

ISSN : 2221-4038

Éditeur

Société Internationale pour l'Histoire du Français Langue Étrangère ou Seconde

Édition imprimée

Date de publication : 1 juin 2015

Pagination : 47-62

ISSN : 0992-7654

Référence électronique

Sergueï Vlassov, « Jean Fleury (1864-1892), auteur d'une grammaire française originale pour les

Russes ", Documents pour l'histoire du français langue étrangère ou seconde [En ligne], 54 | 2015, mis en ligne le 11 février 2021, consulté le 25 mars 2023. URL : http://journals.openedition.org/dhfles/4148 ; DOI : https://doi.org/10.4000/dhfles.4148

Ce document a été généré automatiquement le 25 mars 2023

Tous droits réservés 


\title{
Jean Fleury (1864-1892), auteur d'une grammaire française originale pour les Russes
}

\author{
Sergueï Vlassov
}

1 L'histoire des grammaires du français publiées en Russie reste encore à faire. Pour le XVIII ${ }^{e}$ siècle, quelques rares études (Boulitch 1904, Kouzmina 2001, Kriajeva-Kouzmina 2004, Berelowitch \& Smaguina 2005, Rjéoutski 2005, Vlassov \& Rjéoutski 2013) accordent de l'attention à ce genre de littérature pédagogique ; pour le XIX, très riche en toutes sortes de manuels de français, il n'existe aucun ouvrage d'ensemble. La plupart des chercheurs se limitent à établir la liste des grammaires françaises pour les Russes ou à en analyser brièvement certaines parues au XIX ${ }^{e}$ siècle (AndreevskaïaLevenstern 1953, Andreevskaïa-Levenstern et Mikhaïlova 1958 : 8-48).

2 Sans prétendre combler cette lacune par notre commentaire d'un seul document d'archives, nous souhaitons attirer l'attention sur un Français injustement oublié, auteur d'une grammaire originale pour les Russes.

3 Jean Fleury (1816-1894) est journaliste, poète, linguiste, pédagogue, professeur de langue et de littérature françaises dans plusieurs établissements d'enseignement secondaire et supérieur à Saint-Pétersbourg. Il a enseigné à l'École de droit (appelée parfois en français Collège impérial de jurisprudence) de 1865 à 1883 mais aussi au $5^{\mathrm{e}}$ lycée de garçons de 1865 à 1872, à l'Institut Catherine, à l'Institut Nicolas (cours sur l'histoire universelle de la littérature et celle des beaux-arts), et aux Cours pédagogiques des lycées de filles. Il a été pendant plus de vingt ans lecteur de langue et de littérature françaises à l'université de Saint-Pétersbourg'1.

4 D'autres membres de sa famille ont beaucoup fait pour le rayonnement de la culture française en Russie et de la culture russe en France. Sa deuxième femme, Henriette Alexandrine Fleury (née Léger), était professeur de français et de pédagogie à l'Institut Nicolas (1886-1895), à l'Institut Marie (1882-1889) et à l'Institut de jeunes filles nobles de Saint-Pétersbourg (1890-1891)². La fille de Jean Fleury, Alice, était une femme de lettres bien connue sous son nom de plume Henry Gréville; ses nombreux romans sur 
des sujets russes ${ }^{3}$ ont contribué au regain d'intérêt pour la Russie et sa littérature en France ${ }^{4}$. Le gendre de Jean Fleury, Émile Durand (époux d'Alice) a enseigné lui aussi le français à l'École de droit de Saint-Pétersbourg (1869-1872) et a collaboré pendant vingt ans au Journal de Saint-Pétersbourg en faisant des traductions du russe en français. Avec sa femme, il a fait des traductions d'Ivan Tourguénev et d'Alexandre Ostrovski. On lui doit de belles études (Revue des deux mondes, 15 juin 1876) sur ces écrivains russes et sur le poète ukrainien Taras Chevtchenko ${ }^{5}$.

5 En 1857, Jean Fleury arrive en Russie comme précepteur. La première édition de sa Grammaire en action date de 1864 . Ses trois volumes correspondent à trois parties distinctes. Selon Fleury, la première partie "c'est l'abc de la science», c'est «la classification des mots et l'étude de leurs fonctions dans le discours » (Fleury 1864, II). L'un des côtés novateurs de sa méthode consiste en l'étude systématique de la grammaire non pas à partir de phrases détachées, hors contexte, mais à travers des textes suivis, en "surprenant " les mots et les phrases dans leur contexte ou «en action, et fonctionnant dans le discours, au lieu de les étudier isolés, et à l'état de mort » (Fleury 1864, III). La deuxième partie est l'orthographe, c'est le passage de la forme orale de la langue à sa forme écrite. La troisième "achève l'édifice grammatical», elle aborde la syntaxe «qui s'occupe de l'accord des mots entre eux et de la composition des phrases » (Fleury 1864, II).

6 Fleury commence chaque leçon par une historiette instructive ou plaisante, une anecdote historique, une fable; viennent ensuite des observations et des explications grammaticales suivies d'un exercice sur le texte et d'un nouveau texte, et parfois d'un troisième en supplément. Dans la première édition de La grammaire en action, en tête de la première leçon, l'auteur place une historiette qui fournit des exemples de différentes espèces de mots (verbes, noms, pronoms), puis donne un extrait de «La jeune Sibérienne » de Xavier de Maistre suivi d'exercices sur la définition des parties du discours. Fleury propose à ses élèves de faire la liste des verbes, des noms et des pronoms qui se trouvent dans le texte. Le tout se termine par un questionnaire portant sur les points de grammaire conformes à ses leçons et par des exercices supplémentaires de vocabulaire et de grammaire, par exemple, sur les dérivés des mots étudiés. Ce désir d'ajouter l'agréable à l'utile est caractéristique de la méthode d'enseignement choisie par Fleury.

7 Il utilise des textes suivis même quand il faut remplacer une forme grammaticale par une autre (par exemple, le singulier par le pluriel), ce qui est plus intéressant pour les étudiants confirmés :

I. Formation du pluriel. [Il faut mettre au pluriel les mots soulignés] [...] Dans le pays chaud, le sapajou sautent dans l'acajou, en se faisant des signal ou des grimace; le soir le hibou sortent de leur trou; le corbeau et le freux se nichent dans le rameau des chêne; le chacal, le serval et d'autre animal sauvage, fatal aux être timide et sans défense font retentir l'air de leur cri guttural [...]. (Fleury $1871 \mathrm{a}, 8$ )

8 La dernière partie de cette grammaire est consacrée à la syntaxe et contient un chapitre curieux intitulé «Locutions vicieuses usitées en Russie». À notre connaissance, Fleury est le premier à proposer des exercices qui portent sur les fautes les plus typiques des Russes ${ }^{6}$. Ils restent toujours d'actualité.

9 La méthode de Fleury est une variété des méthodes basées sur la lecture du texte ${ }^{7}$. Fleury ne veut pas s'ériger en un pédant qui légifère en expliquant aux élèves les lois de la grammaire. Il veut éveiller l'intérêt des élèves pour la langue, en leur proposant des 
textes amusants, touchants ou instructifs et en les invitant à observer eux-mêmes les faits grammaticaux dans les textes. Cela exige de leur part une attention continue et leur permet de retenir aisément les textes avec le vocabulaire et la théorie grammaticale qui s'y rapportent. Nous avons ici un procédé mnémotechnique efficace qui a recours à la mémoire, aux sentiments et à la capacité d'observation, de jugement et de réflexion des élèves.

10 La première et la quatrième édition de la Grammaire en action de Jean Fleury ont été autorisées par le Comité scientifique du ministère russe de l'Instruction publique en tant que manuel facultatif "pour les classes moyennes et supérieures de tous les établissements d'instruction ${ }^{8} »$. Dans les éditions postérieures ${ }^{9}$, Fleury est obligé d'adapter son ouvrage à son enseignement à l'université : la théorie grammaticale y est présentée séparément et précède les exercices pratiques. Cette présentation correspondait probablement davantage à l'organisation traditionnelle de l'enseignement des langues dans lequel l'exposition de la théorie grammaticale précédait les cours pratiques de grammaire.

11 Le document d'archives que nous publions est une lettre de Fleury du 8 novembre 1885 adressée au président du Comité scientifique du ministère de l'Instruction publique, Alexandre Guéorguievski. Fleury y parle du refus du Comité de faire figurer la sixième édition de sa Grammaire en action parmi les manuels et les посовиЕ (livres auxiliaires) autorisés.

Le lecteur français expose ses idées sur l'enseignement de la langue française aux Russes. À l'en suivre, les grammaires de Chapsal et de Larousse, destinées aux Français, ne conviennent pas à l'enseignement de la grammaire française aux étrangers. Fleury préconise la méthode comparée qui vise à relever des différences grammaticales et lexicales entre le russe et le français. Il souligne son apport non seulement aux méthodes d'enseignement du français aux Russes, mais aussi sa contribution à l'amélioration de la description grammaticale du français, notamment l'emploi des temps passés et des modes, l'ordre des mots et les règles de l'emploi du participe passé. Le caractère novateur de la quatrième édition de sa grammaire lui a valu les suffrages des grands savants français, tels que Michel Bréal et Arsène Darmesteter, et son admission à la Société linguistique de Paris.

La lettre de Fleury nous est parvenue grâce à une copie signée par lui et faite à l'intention de son collègue de l'université de Saint-Pétersbourg, Ivan Pomialovski, professeur de lettres classiques et membre du Comité scientifique ${ }^{10}$. Cette copie est jointe à deux billets autographes de Fleury. Dans le premier mot non daté, Fleury prie Ivan Pomialovski d'intervenir en faveur de sa grammaire auprès du Comité scientifique. Il fait remarquer qu'il est « chargé par le ministère de l'Instruction publique de France de faire un travail sur l'administration de l'Instruction publique en Russie, rapport qui sera imprimé » $\left(f^{\circ} 2\right)$. Il dit vouloir éviter d'être «de mauvaise humeur » contre le Comité scientifique "qui adopte tant de livres en français déplorable ", "et qui ferme impitoyablement la porte» au sien, "lequel peut avoir des défauts, mais qui est un travail original et savant, et non une simple adaptation de ce qui existe » ( $\left.f^{\circ} 2-2 v\right)$. Dans la deuxième petite lettre, datée du 17 décembre 1885, également adresséE à Pomialovski, Fleury renchérit: si le Comité ne fait pas justice à sa demande, il a l'intention de faire un "délicieux pamphlet», en reproduisant le rapport contre sa Grammaire dont il avait «pris copie » (f॰ $3-4)$. Au fait, Fleury se joue des fonctionnaires russes car ils craignent l'opinion publique en Occident. Il observe tout de suite : «Je 
n'en ferai rien si l'on me rend justice, mais je veux avoir une arme pour le cas contraire. Tout ceci entre nous, bien entendu [...] $»\left(f^{\circ} 4\right)$.

Le Comité scientifique ne semble pas avoir donné suite à son refus d'autoriser la grammaire de Fleury, car celle-ci a eu encore trois éditions dont la dernière (la $9^{\mathrm{e}}$ ) a été publiée en 1892.

En plus du manuel et des exercices de grammaire, Jean Fleury a publié plusieurs travaux de linguistique et de dialectologie française, dont certains sont parus en Russie (Fleury 1873 ; Fleury 1879 ; Fleury [1885] ; Fleury 1890 ; Fleury 1891 ; Fleury 1893). C'est l'un des promoteurs des recherches scientifiques sur la langue française en Russie ${ }^{11}$ et l'un des premiers, sinon le premier, à nous donner des études originales sur la langue française comparée au russe.

[Lettre de Jean Fleury du 8 novembre 1885 à Alexandre Guéorguievski ${ }^{12}$, président du Comité scientifique du ministère de l'Instruction publique]

Copie envoyée au professeur Ivan Pomialovski, membre du Comité scientifique du ministère de l'Instruction publique - $\mathrm{f}^{\circ}$ 5-6.

18 À Son Excellence Monsieur le Président du Comité scientifique du ministère de l'Instruction publique

19 Excellence [,]

Permettez-moi d'en appeler de la sentence du Comité scientifique qui m'a été signifiée le 2 novembre au sujet de ma Grammaire en action $6^{\mathrm{e}}$ édition.

D'abord, cette décision, je ne l'ai pas sollicitée, et le Comité, si je ne me trompe, ne donne son jugement sur un livre que si l'auteur l'en a prié.

La première édition de ma Grammaire était toute française [Fleury 1864]. Elle se composait de trois volumes et justifiait son titre de Grammaire en action par la méthode qui y était employée. Cette méthode exigeant de longs développements et supposant des élèves étrangers à toute notion grammaticale, j'ai dû y renoncer quand il a fallu m'adresser à des enfants déjà un peu instruits, mais j'ai cru pouvoir sans inconvénient conserver le titre qui m'avait si bien servi. Cette première édition, soumise au Comité scientifique, fut autorisée par lui comme посовиЕ ${ }^{13}$ pour les classes moyennes en Russie.

23 La quatrième édition [Fleury 1876 a.], réduite à un petit volume avec traduction russe fut autorisée dans les mêmes conditions par le Comité scientifique. Je fus informé de cette décision par un avis en date du 22 septembre 1876.

24 La sixième édition ${ }^{14}$ n'étant guère que la reproduction de la quatrième, je n'ai pas cru avoir à solliciter un nouvel examen. Les quelques améliorations que j'y ai apportées ne s'attachent qu'à des détails. Il y a peut-être quelques fautes d'impression de plus. Mais quel livre imprimé en Russie est exempt de ces fautes? C'est un défaut qu'il est d'ailleurs facile de faire disparaitre en réimprimant les pages fautives, en faisant ce qu'en termes d'imprimerie on appelle des cartons.

Secondement, je n'ai jamais demandé et je ne demande pas que ma Grammaire soit imposée comme Manuel obligatoire. Il est bien évident que les maîtres qui arrivent de France imprégnés de Chapsal [Noël \& Chapsal 1878, Noël \& Chapsal 1853] et de Larousse [Larousse 1849-1868] seront peu disposés à se remettre à l'étude pour enseigner d'après ma méthode. Si cette méthode prend le dessus, ce ne pourra être que par degrés. 
26 Toutes les grammaires que je connais sont faites pour les Français, j'en ai voulu faire une pour les Russes, une grammaire dans laquelle on passe de la langue russe, grammaticalement étudiée, à la langue française. C'est en faisant traduire du russe en français pendant vingt années d'enseignement que j'ai senti l'insuffisance des grammaires ordinaires et que d'observation en observation j'ai formulée la mienne.

27 J'ai constaté que ce qui embarrassait le plus les Russes dans ce travail, c'était l'emploi des temps passés - l'ordre des mots dans la phrase - les cas où il faut employer le subjonctif. J'ai réussi à trouver pour ces difficultés des règles simples et exprimées en quelques mots et sans exceptions tant qu'on ne torture pas la phrase française. Ce sont là trois points capitaux de mon livre. J'ai étudié aussi, systématiquement, l'orthographe usuelle, j'ai réduit à une seule les règles du participe passé. J'ai pris soin aussi de reléguer sur le second plan tous les détails secondaires.

28 Que les maîtres qui arrivent de France et ne connaissent pas la langue russe grammaticalement, soient peu sensibles à ces innovations, cela se conçoit, mais il n'en est pas partout. Je reçois à chaque instant des lettres de l'intérieur dans lesquelles les maîtres se félicitent des progrès qu'ils ont provoqués à l'aide de ma grammaire dans le milieu tout russe où ils vivent, et me pressent d'obtenir l'insertion du titre de mon livre dans la liste annuelle par le Comité scientifique des Manuels et des посоБія autorisés. C'est pour satisfaire à ces réclamations que je me suis adressé au Comité scientifique et non pour provoquer un examen dont je croyais pouvoir me passer.

Cet examen, je le réclamerai plus tard, lorsque mon travail sera définitif. Mais je prierai alors le Comité de remarquer que sous sa forme modeste, ma Grammaire est un livre savant, qui demande à être jugé non par de simples praticiens, mais par des linguistes. Il ne s'agit pas d'examiner jusqu'à quel point je me suis écarté de la routine, mais quand j'ai eu raison de m'en écarter.

30 Votre Excellence a pu voir dans ma Préface que le suffrage des hommes compétents ${ }^{15}$ 'a pas manqué à ma Grammaire et ces approbations ne sont certes pas des œuvres de complaisance. Je n'ai vu $\mathrm{M}$. Arsène Darmesteter qu'une seule fois en ma vie, et je n'ai jamais vu M. Michel Bréal. Ces messieurs ne me connaissent que par mes écrits. C'est la quatrième édition de cette même Grammaire qui m'a valu d'être admis dans la Société de linguistique de Paris, qui place dans ses Mémoires les travaux que je lui envoie côte à côte avec ceux des princes de la science. Quelques-uns de mes articles ont été tirés à part et je me permets de joindre à cette lettre un de ces tirages.

31 Quoique le nombre des livres qui paraissent en France soit énorme, chaque fois que je publie un ouvrage, toute la presse s'en occupe, non seulement en France, en Belgique, en Suisse, mais en Angleterre, en Allemagne, en Italie. Je pourrais faire un beau volume des articles élogieux qui m'ont été consacrés dans toutes les langues par des mains inconnues. J'ai adressé un de mes ouvrages à l'Académie française [Fleury 1876 b.], elle m'a accordé une première mention honorable en me promettant de faire mieux à un prochain envoi. Mon travail en deux volumes sur Rabelais a été abrégé en un volume en anglais et en italien. Mon Histoire élémentaire de la littérature française [Fleury 1871b.] est employée comme Manuel dans une foule d'établissements dans tous les pays de l'Europe et même aux États-Unis. Ce qu'on loue surtout dans mon style c'est la clarté, la précision qui dit tout en peu de mots. Il me semble que cette situation que je me suis faite au dehors par mes écrits méritait bien quelques égards, qu'elle aurait dû me défendre contre certaines attaques, certaines insinuations, et que lors même que ma Grammaire serait défectueuse - ce que je suis loin d'admettre - on n'aurait pas dû lui 
fermer brutalement la porte et la placer par conséquent au-dessous de maints livres écrits dans un français impossible et qui obtiennent un laisser-passer.

Permettez-moi donc d'espérer, Monsieur le Président, que le Comité voudra bien revenir sur sa décision du 2 novembre - soit en l'annulant purement et simplement et me laissant bénéficier de la décision de 1876 - soit en autorisant à nouveau ma Grammaire commе посовіе. Je ferai remarquer d'ailleurs que la décision du Comité scientifique au sujet de ma sixième édition est caduque. Cette édition est épuisée depuis quelques mois. J'en prépare une nouvelle où je tiendrai compte de celles des observations qu'on m'a faites, qui me sembleront fondées.

Je prie Votre Excellence d'agréer l'assurance de ma haute estime et de mon profond respect.

Jean Fleury

Auteur de Rabelais et ses ceuvres. 2v. In $8^{\circ}$. Marivaux et le Marivaudage ${ }^{16} .1 \mathrm{v}$. in $8^{\circ}$. Histoire élémentaire de la littérature française $2 \mathrm{v}$. in 12 . Krylof et ses fables ${ }^{17}$ in 12 . Littérature orale de la Basse Normandie ${ }^{18}, 1 \mathrm{v}$. in 16, de diverses brochures et de nombreux ouvrages imprimés en Russie et ailleurs Saint-Pétersbourg 8 novembre 1885

Collaborateur ordinaire de Romania, de la Bibliothèque universelle suisse, de la Revue Internationale (Florence), de la Revue savoisienne etc. des Mémoires de la Société de linguistique de Paris, du J[ournal] d[e] Saint-Pétersbourg, ayant fourni nombre d'articles à la Revue politique et littéraire, à la Nouvelle Revue, etc.

\section{BIBLIOGRAPHIE}

\section{Sources primaires}

\section{Documents d'archives}

Bibliothèque nationale de Russie, Mss, fonds 608 (archives de I. V. Pomialovski), op. 1, d. 1372.

Archives centrales historiques de Saint-Pétersbourg (TSGIA), fonds 2, op. 1, d. 13431, fonds 10, op. 1, d. 3424.

\section{Sources imprimées}

ANGUELOV, Iouri (1852). ВСПОМОГАТЕЛЬНОЕ СРЕДСТВО ДЛЯ УЧАЩИХСЯ ФРАНЦУЗСКОМУ ЯЗЫКУ ПО МЕТОДЕ ЖАКОТо [Iouri Anguelov, Manuel auxiliaire pour ceux qui apprennent le français d'après la méthode Jacotot]. Saint-Pétersbourg : D. Quesneville.

BASTIN, Jean (1879-1879). Étude philologique de la langue française ou Grammaire comparée et basée sur le latin. Saint-Pétersbourg.

BASTIN, Jean (1880). Le participe passé dans la langue française et son histoire. Saint-Pétersbourg (la $2^{\mathrm{e}}$ et la $3^{\mathrm{e}}$ éd. portent le titre : Étude des participes basée sur l'histoire de la langue. Saint-Pétersbourg, 1888 et 1889 ). 
BASTIN, Jean (1891). Étude sur les principaux adverbes. Affirmation, négation, manière. Paris : E. Capiomont.

BASTIN, Jean (1896). Le verbe et les principaux adverbes dans la langue française : étude historique. Saint-Pétersbourg : Trenké.

BOUGEAULT, Alfred (1858). Difficultés et finesses de la langue française. Seconde édition. SaintPétersbourg : S. Dufour [1 ${ }^{\text {re }}$ éd., Saint-Pétersbourg : Clusel, Belizard, 1853].

CONSTANTIN, Aimé (1859-1860). КОНСТАНТЕН, ЭМЭ. ПОЛНЫЙ КУРС ФРАНЦУЗСКОГО ЯЗЫКА, ПО МЕТОДЕ РОБЕРТСОНА [Cours complet de langue française d'après la méthode Robertson]. SaintPétersbourg : A. Jakobson.

DIEMER, Charles (1847). La langue française enseignée aux Russes d'après la méthode Robertson. SaintPétersbourg, Charles Kray ( $2^{\mathrm{e}}$ édition, entièrement refondue, corrigée et augmentée par Auguste Boltz, Berlin, C. Schultze, Saint-Pétersbourg, Jacques Issakoff [sic], 1855).

[FÉNELON, François] (1832). Aventures de Télémaque, à l'usage des élèves de l'enseignement universel, dans le Corps de la Marine. Saint-Pétersbourg : Pluchart.

FLEURY, Jean (1864). La Grammaire en action. 3 vol. Paris : C. Borrani.

FLEURY, Jean (1869). Krylov et ses fables. Paris : Hachette, 1869.

FLEURY, Jean (1871a). Grammaire russe-française. Saint-Pétersbourg/Moscou.

FLEURY, Jean (1871b). Histoire élémentaire de la littérature française. Saint-Pétersbourg/Moscou, ( $2^{\mathrm{e}}$ éd., Paris, Plon; Saint-Pétersbourg : chez l'auteur, 1879 ; $9^{\mathrm{e}}$ éd., Paris : Plon ; Saint-Pétersbourg, 1894).

FLEURY, Jean (1873). Du caractère spécial de la langue et de la littérature française. Saint-Pétersbourg. FLEURY, Jean (1876 a). La Grammaire en action, 4 e édition. Considérablement simplifiée et accompagnée d'une traduction en russe. Saint-Pétersbourg : chez les principaux libraires.

FLEURY, Jean (1876 b). Rabelais et son œuvre. 2 vol. Paris : Didier et Cie.

FLEURY, Jean (1879). Un peuple retrouvé par la grammaire. Notions élémentaires de linguistique. Paris : F. Vieweg.

FLEURY, Jean (1881). Marivaux et le marivaudage. Paris : E. Plon.

FLEURY, Jean (1883). Littérature orale de la Basse-Normandie. Paris : Maisonneuve.

FLEURY, Jean (1885). « Essai sur le patois normand de la Hague », tiré-à-part des Mémoires de la Société de linguistique de Paris, [1885], t. 5 (idem, Paris, 1886).

FLEURY, Jean (1890). Les aspects des verbes russes et les temps des verbes français. Saint-Pétersbourg. FLEURY, Jean (1891). La Presqu'île de la Manche et l'Archipel anglo-normand. Essai sur le patois de ce pays. Supplément à l'Essai sur le patois normand de La Hague. Paris : Jean Maisonneuve.

FLEURY, Jean (1893). Les aspects et les temps : La conjugaison russe et la conjugaison du français et des autres langues romanes comparées. Saint-Pétersbourg: Trenké et Fusnot.

FLEURY, Jean (1894). Histoire élémentaire de la littérature française depuis l'origine jusqu'à nos jours. $9^{\mathrm{e}}$ éd. Paris : E. Plon, Nourrit ; Saint-Pétersbourg : chez l'auteur.

KLENTZE, Bernard (1893-1897). КУРС ФРАНЦУЗСКОГО ЯЗЫКА В ТРЕХ ЧАСТЯХ [Bernard Klentze, Cours de langue française en trois parties]. Moscou : V. V. Doumnov. 
LAROUSSE, Pierre (1848). La lexicologie des écoles. Cours complet de langue française et de style divisé en 3 années. Paris, plusieurs éditions chez Maire-Nyon, Boyer et Larousse depuis 1849-1868 jusqu'au début du XXe siècle.

NOËL, François-Joseph-Michel \& CHAPSAL, Charles Pierre (1853, $2^{\mathrm{e}}$ éd.). Grammaire française de Noël et Chapsal, avec la traduction russe en regard. Saint-Pétersbourg : Stépane Gouro.

NOËL, François-Joseph-Michel \& CHAPSAL, Charles Pierre (1878). Nouvelle Grammaire française sur un plan très méthodique, avec de nombreuses exercices d'ort[h]ographe, de syntaxe et de ponctuation [...]. Saint-Pétersbourg/Moscou : Wolff.

\section{Sources secondaires}

ALEXEEV, Mikhaïl (1948). « И. С. ТУРГЕНЕВ - ПРОПАГАНДИСТ РУССКОЙ ЛИТЕРАТУРЫ НА ЗАПАДЕ ». АН СССР. ИНСТИТУТ ЛИТЕРАТУРЫ (ПУШКИНСКИЙ ДОМ). ТРУДЫ ОТДЕЛА НОВОЙ РУССКОЙ ЛИТЕРАТУРЫ [« I. S. Tourguénev, propagandiste de la littérature russe en Occident ». Institut de littérature (Maison Pouchkine) de l'Académie des sciences de l'URSS, Travaux de la section de nouvelle littérature russe]. t. 1. Moscou/Léningrad, 39-80.

ANDREEVSKAIA-LEVENSTERN, Lydia (1953). ИСТОРИЯ МЕТОДИЧЕСКИХ НАПРАВЛЕНИЙ В ОБУЧЕНИИ В РОССИИ ВО 2-ОЙ ПОЛОВИНЕ ХІХ ВЕКА (60-80 ГГ.) И ОТРАЖЕНИЕ ИХ В УЧЕБНОЙ ЛИТЕРАТУРЕ. [Histoire des courants didactiques dans l'enseignement en Russie dans la deuxième moitié du XIX siècle et leur reflet dans les livres d'études (résumé de thèse)]. Moscou : Institut pédagogique d'État.

ANDREEVSKAIA-LEVENSTERN Lydia \& MIKHAILOVA, Olga (1958). МЕТОДИКА ПРЕПОДАВАНИЯ ФРАНЦУЗСКОГО ЯЗЫКА В СРЕДНЕЙ ШКОЛЕ [Méthodologie de l'enseignement du français à l'école secondaire]. Moscou : Outchpedguiz.

BARBEY D'AUREVILLY, Jules-Amédée (1878). Les œuvres et les hommes. $5^{\mathrm{e}}$ partie. « Les bas bleus ». ch. XXIII. Henry Gréville. Paris :Victor Palmey \& Bruxelles : G. Lebrocquy, 293-302.

BERELOWITCH, Wladimir \& SMAGUINA, Galina (2005). « Enseignants et modèles éducatifs français à Saint-Pétersbourg au XVIII ${ }^{\mathrm{e}}$ siècle ». In La France et les Français à Saint-Pétersbourg, XVIII ${ }^{e}$ ХХ⿰㇒ siècles. САНКТ-ПЕТЕРБУРГ, ЕВРОПЕЙСКИЙ ДОМ [Saint-Pétersbourg : Evropejskij Dom], 36-69.

BOULITCH, Sergueï (1904). очЕРКИ ИСТОРИИ ЯЗЫКОЗНАНИЯ В РОссии [Aperçu de l'histoire de la linguistique en Russie]. Vol. I (XIII' ${ }^{\mathrm{e}}$ s. - 1825). Saint-Pétersbourg : Tipografiia M. Merkoucheva. DICTIONNAIRE DE BIOGRAPHIE FRANÇOISE (1970).T. 12. Paris : Letouzey et Ané.

(LA) GRANDE ENCYCLOPEDIE (1885-1902). 31 vol. Paris : H. Lamiraut, Société anonyme de La Grande Encyclopédie. t. XV.

KARTSOV, NicolaÏ (1897). (ИСТОРИЧЕСКИЙ ОЧЕРК). САНКТ-ПЕТЕРБУРГ, ТИПОГРАФИЯ САНКТПЕТЕРБУРГСКОГО ГРАДОНАЧАЛЬСТВА [L'Institut Marie 1797-1897 (Aperçu historique). SaintPétersbourg : Imprimerie du gouvernement de Saint-Pétersbourg].

KOUZMINA, Nadejda (2000). « ПЕРВЫЕ УЧЕБНЫЕ ПОСОБИЯ ПО ФРАНЦУЗСКОМУ ЯЗЫКУ, ИЗДАННЫЕ В РОССИИ », ВЕСТНИК МОСКОВСКОГО УНИВЕРСИТЕТА, СЕР. 19 ЛИНГВИСТИКА И МЕЖКУЛЬТУРНАЯ КомМУНИКАЦИЯ [« Les premiers manuels de français édités en Russie ». Messager de l'université de Moscou. Série 19 - Linguistique et communication interculturelle]. $\mathrm{n}^{\circ}$ 1, 20-29.

KRIAJEVA-KOUZMINA, Nadejda (2004). Description de la langue russe par les auteurs français de la seconde moitié du XVIII ${ }^{e}$ siècle, ch. 3, Thèse de doctorat de l'Université de Nancy 2.519 pages. http://docnum.univ-lorraine.fr/public/NANCY2/doc205/2004NAN21011.pdf.

LEGER, Louis (1880). « Le roman russe dans la littérature française. Madame Henri Gréville », Nouvelles études slaves. Histoire et littérature, 1880, 107-140. 
MAKACHINE, SergueÏ (1937). « ЛИТЕРАТУРНЫЕ ВЗАИМООТНОШЕНИЯ РОССИИ И ФРАНЦИИ XVIIIХІХ ВВ », ЛИТЕРАТУРНОЕ НАСЛЕДСТВО, ВЫП. 29-30 [« Les rapports littéraires de la Russie et de la France aux XVIII ${ }^{\mathrm{e}}$-XIX ${ }^{\mathrm{e}}$ siècles », L'héritage littéraire, fasc. 29-30], Moscou : Journalnogazetnoéob'edinenié, V-LXXXII.

MIKHEEVA, Anna (1999). « РУССКИЕ » РОМАНЫ ВО ФРАНЦУЗСКОЙ ЛИТЕРАТУРЕ : ‘ИСКУПЛЕНИЕ САВЕЛИЯ’ И ‘ДОЗЯ’ АНРИ ГРЕВИЛЬ, ІП ВЗАИМОСВЯЗИ И ВЗАИМОВЛИЯНИЕ РУССКОЙ И ЕВРОПЕЙСКОЙ ЛИТЕРАТУР. МАТЕРИАЛЫ МЕЖДУНАРОДНОЙ КОНФЕРЕНЦИИ [« Les romans 'russes' dans la littérature française : 'L'expiation de Savéli' et 'Dosia' d'Henry Gréville ». Les rapports et l'influence réciproques de la littérature russe et européenne. Les actes du colloque international. SaintPétersbourg 13-15 novembre 1997. Saint-Pétersbourg : éditions de l'Université de SaintPétersbourg, 227-231.

RJÉOUTSKI, Vladislav (2005). « Les écoles étrangères dans la société russe à l'époque des Lumières ». Cahiers du Monde russe, 46/3 (juillet-septembre), 473-528.

TIMOFEEV, Vladimir (1887). С.-ПЕТЕРБУРГСКОГО НИКОЛАЕВСКОГО СИРОТСКОГО ИНСТИТУТА. САНКТПЕТЕРБУРГ, ЭКСПЕДИЦИЯ ЗАГОТОВЛЕНИЯ ГОСУДАРСТВЕННЫХ БУМАГ [Le 50 anniversaire de

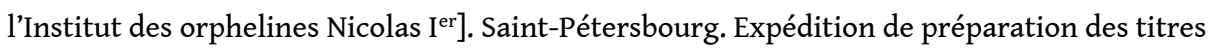
d'État. Annexes : Liste des personnes en poste aux établissements d'orphelines, $\left.\mathrm{n}^{\circ} 50\right]$.

VLASSOV, Sergueï \& RJÉOUTSKI, Vladislav (2013). « L'enseignement de la grammaire française en Russie : enseignants, méthodes et livres utilisés ». Documents pour l'histoire du français langue étrangère ou seconde, $51 \mid 2013$ [Gisèle Kahn \& Nadia Minerva (dir.). Grammaire et enseignement du français langue étrangère et seconde - Permanences et ruptures du XVI ${ }^{e}$ au milieu du XXe siècle], 105-128.

VLASSOV, Sergueï. «Jean Fleury, lecteur de langue et de littérature française à l'université de Saint-Pétersbourg (1872-1894) ». À paraître dans Francine-Dominique Liechtenhan \& Vladislav Rjéoutski (dir.). Actes du colloque « Les Français dans la vie intellectuelle et scientifique en Russie au XIX ${ }^{e}$ siècle », Paris : fondation Singer-Polignac, (16-17 septembre 2011).

\section{NOTES}

1. Pour plus de détails, voir Sergueï Vlassov, «Jean Fleury, lecteur de langue et de littérature françaises à l'Université de Saint-Pétersbourg (1872-1894) », à paraître dans les Actes du colloque «Les Français dans la vie intellectuelle et scientifique en Russie au XIXe siècle », Paris, Fondation SingerPolignac, les 16 et 17 septembre 2011, sous la dir. de Francine-Dominique Liechtenhan et Vladislav Rjéoutski.

2. Sur Henriette Fleury, épouse de Jean Fleury, Timofeev 1887 ; Annexes : Liste des personnes en poste aux établissements d'orphelines, $n^{\circ} 50$, Kartsov 1897, 15 ; Archives centrales historiques de Saint-Pétersbourg, fonds 2, op. 1, d. 13431 ; fonds 10, op. 1, d. 3424).

3. Citons parmi les plus connus Dosia, La princesse Oghérof, La niania, L'Expiation de Savéli, Les Koumiassine.

4. Sur Alice Fleury - Durand-Gréville : «Durand-Gréville (Alice-Marie-Céleste Henry, Madame Émile) ", Dictionnaire de biographie française 1970, t. 12, col. 694-695; «Durand-Gréville (Alice Fleury, femme)», La Grande Encyclopédie [1885-1902], t. XV, 114; Barbey d'Aurevilly 1878, 293-302 ; Leger 1880, 107-140 «Le roman russe dans la littérature française. Madame Henri Gréville »; Fleury 1894, 471-472 ; cf. Makachine 1937 : LXIV ; Mikhéeva 1999.

5. Sur Émile Durand : «Durand-Gréville (Émile-Alix)», Dictionnaire de biographie française 1970, t. 12, 1970, col. 695 ; «Durand-Gréville (Émile-Alix) », La Grande Encyclopédie [1885-1902], t. XV, 113) ; Alexeev 1948, 66-67, 69. 
6. Cf. Bougeault 1858, où l'on trouve déjà un « Recueil de Locutions vicieuses usitées en Russie » (p. 1-11), mais sans exercices.

7. Voyez, par exemple les ouvrages [Fénelon] (1832); Anguelov (1852); Diemer (1847); Constantin (1859-1860); Klentze (1893-1897).

8. Cf. page de titre de Fleury 1892 ; voir aussi le début de la lettre publiée ci-dessus selon laquelle la grammaire de Fleury était autorisée avant tout pour les classes moyennes.

9. La grammaire de Fleury a connu neuf éditions de 1864 à 1892.

10. Bibliothèque nationale de Russie, Mss, fonds 608 (archives de I. V. Pomialovski), op. 1, d. 1372. Pomialovski, Ivan Vassilievitch (1845-1906), historien, philologue et archéologue russe, professeur de lettres classiques à l'université de Saint-Pétersbourg, membre du Conseil du ministre de l'Instruction publique et du Comité scientifique du ministère de l'Instruction publique. Nous indiquons plus loin les feuillets de cette lettre dans le corps du texte.

11. Il faut signaler aussi les travaux de Jean Bastin, un collègue de Jean Fleury à l'École Impériale de droit de Saint-Pétersbourg, devenu son ennemi et un concurrent déloyal qui a essayé, mais en vain, de dénigrer Fleury aux yeux de l'administration de l'université de Saint-Pétersbourg lors du concours pour le poste vacant de lecteur en langue française annoncé par la faculté historicophilologique de l'université en 1872 (Bastin 1878-1879, Bastin 1880, Bastin 1891, Bastin 1896, etc.). 12. Guéorguievski, Alexandre Ivanovitch (1830-1911), haut fonctionnaire du ministère de l'Instruction publique en Russie, directeur de la Revue du ministère de l'Instruction publique en 1866-1881.

13. Manuel facultatif, livre auxiliaire.

14. La 6e édition de la grammaire de Fleury qui doit dater de 1885 est absente des bibliothèques de Saint-Pétersbourg.

15. Il s'agit des avis d'Arsène Darmesteter et de Michel Bréal sur la grammaire de Jean Fleury insérés dans la Préface de la Grammaire en action.

16. (Fleury 1881).

17. (Fleury 1869).

18. (Fleury 1883).

\section{RÉSUMÉS}

L'article traite des méthodes d'enseignement du français introduites en Russie par Jean Fleury, lecteur de langue et de littérature françaises à l'Université de Saint-Pétersbourg de 1872 à 1894 . Fleury préconise la méthode comparée qui vise à relever des différences grammaticales et lexicales entre le russe et le français. En plus de «La Grammaire en action » (plusieurs éditions et remaniements depuis 1864 jusqu'à 1892), Jean Fleury a publié plusieurs travaux de linguistique et de dialectologie française, dont certains sont parus en Russie. C'est l'un des promoteurs des recherches scientifiques sur la langue française en Russie et l'un des premiers, sinon le premier, à nous donner des études originales sur la langue française comparée au russe. L'article est suivi d'une lettre de J. Fleury en date du 8 novembre 1885 adressée au président du Comité scientifique du Ministère de l'Instruction publique, Alexandre Guéorguievski. Dans cette lettre l'éminent pédagogue français, injustement oublié aujourd'hui, souligne son apport non seulement aux méthodes d'enseignement du français aux Russes, mais aussi sa contribution à l'amélioration de la description grammaticale du français en comparaison du russe, notamment en ce qui concerne 
l'emploi des temps passés et des modes, l'ordre des mots et les règles de l'emploi du participe passé.

The article deals with methods of teaching French introduced in Russia by Jean Fleury, lecturer of French language and French literature at the University of St. Petersburg from 1872 to 1894. Fleury advocates comparative method that aims to identify grammatical and lexical differences between Russian and French. In addition to "Grammar in Action" (several editions and revisions since 1864 until 1892), Jean Fleury has published several works of French linguistics and dialectology, some of which have appeared in Russia. This is one of the promoters of scientific research on the French language in Russia and one of the first, if not the first, to give us the original studies on the French language compared to Russian. The article is followed by a letter of J. Fleury dated November 8, 1885 to the Chairman of the Scientific Committee of the Ministry of Education, Alexander Guéorguievski. In this letter the eminent French pedagogue unjustly forgotten today, emphasizes his contribution not only to the teaching methods of the French to the Russians, but also his contribution to the improvement of the grammatical description of the French compared to the Russian, including the use of past tenses and moods, the word order and the rules of the use of the past participle.

\section{INDEX}

Keywords : Jean Fleury, University of St. Petersburg, teaching French grammar, grammatical description of the French, comparative study of French and Russian, grammatical and lexical differences between Russian and French

Mots-clés : Jean Fleury, université de Saint-Pétersbourg, enseignement de la grammaire française, description grammaticale du français, étude comparée du français et du russe, différences grammaticales et lexicales entre le russe et le français

\section{AUTEUR}

\section{SERGUEÏ VLASSOV}

Université de Saint-Pétersbourg

vlasovsv7@gmail.com 